\title{
Conscience phonologique et construction identitaire d'élèves de cycle trois
}

Sophie Briquet-Duhazé

\section{(2) OpenEdition}

1 Journals

Édition électronique

URL : http://journals.openedition.org/trema/2551

DOI : $10.4000 /$ trema. 2551

ISSN : 2107-0997

Éditeur

Faculté d'Éducation de l'université de Montpellier

\section{Édition imprimée}

Date de publication : 1 décembre 2010

Pagination : 73 - 87

ISSN : 1167-315X

\section{Référence électronique}

Sophie Briquet-Duhazé, « Conscience phonologique et construction identitaire d'élèves de cycle trois », Tréma [En ligne], 33 - 34 | 2010, mis en ligne le 01 décembre 2012, consulté le 01 mai 2019. URL :

http://journals.openedition.org/trema/2551 ; DOI : 10.4000/trema.2551

Ce document a été généré automatiquement le 1 mai 2019.

Trema 


\title{
Conscience phonologique et construction identitaire d'élèves de cycle trois
}

\author{
Sophie Briquet-Duhazé
}

\section{Introduction}

1 Une société donnée, qui possède ses propres règles, participe à construire l'identité d'un enfant. Sa socialisation intègre les caractéristiques locales, professionnelles, culturelles... de sa famille. Puis, ses expériences sociales vont contribuer à enrichir et transformer son identité. A l'école, ses performances scolaires, notamment celles réalisées dans et par l'apprentissage de la lecture, concourent également à construire, développer et modifier son identité en lien avec celle dont il a hérité. La trajectoire de l'élève peut être marquée par un " apprentissage de la lecture raté » selon l'expression de NONNON et GOIGOUX (2007) ${ }^{1}$ . C'est une remise en cause de l'identité en construction, que les média, les évaluations nationales et internationales ne cessent de véhiculer, rapporter sur le long terme et dont l'école ne sait si elle doit se sentir coupable ou victime. Le modèle spatio-temporel (repris par AYRAUD et GUIBERT, 2000) joue alors un rôle plus que négatif (l'institution reproduit dans la durée le fait de ne pas réussir à apprendre à lire à un élève) là où ce triptyque permet à d'autres de jouer un rôle déterminant dans les processus de transaction relationnelle et biographique (DUBAR, 1994) et donc dans leur développement identitaire au coeur de la microsociété qu'est l'école.

2 La recherche que nous proposons d'exposer vise à analyser si une catégorie précise et délimitée de difficultés peut être repérée et identifiée comme étant un marqueur individuel et collectif important de l'échec persistant en lecture au cycle trois. Côté élève, il s'agit précisément, au-delà de l'approche globale de ses difficultés, de lui permettre de travailler conjointement sur la restauration de son image et du lien social sans différenciation des remédiations apportées au sein de la classe entière. 
3 Dans un premier temps, nous annoncerons la problématique et le cadre théorique. Puis nous présenterons les choix méthodologiques, notamment les évaluations des élèves et le travail avec les maîtres. Enfin, les résultats seront développés et discutés du point de vue de la construction identitaire en nous référant aux entretiens avec les enseignants lors de la présentation initiale et finale des résultats de leurs élèves.

\section{Problématique}

4 La conscience phonologique, qui est la connaissance consciente que les mots du langage oral sont composés d'unités plus petites, apparaît comme un bon prédicteur, au début du $\mathrm{CP}$, d'un apprentissage de la lecture réussi si tant est qu'il est progressif, régulier et continu. Un deuxième prédicteur serait la connaissance des lettres dans ses trois valeurs (leur nom, le son qu'elles produisent, leur graphie).

Cependant, qu'en est-il lorsque le cycle deux est achevé (fin de CE1) et que l'apprentissage de la lecture est peu ou pas acquis? Depuis les années 1980, le concept d'échec scolaire, défini à la suite d'enquêtes montrant combien les élèves issus des milieux les plus défavorisés échouent quelquefois dans des proportions importantes, a été le point de départ de réformes diverses, dont la lutte contre l'illettrisme. Mais le sentiment d'incapacité à sortir de ce pourcentage d'élèves ne maîtrisant pas les savoirs de base à l'entrée en sixième demeure et génère, pour le moins, une culpabilité collective. Elèves, parents, enseignants et plus largement institutions, partagent le poids de cet échec là où chacun tente d'y faire naître la réussite. Notre problématique s'inscrit donc dans ce contexte des $15 \%$ d'élèves en grande difficulté à la fin du primaire, en cherchant à revisiter une acceptation scientifique : celle qui consiste à considérer que la conscience phonologique et son entraînement ont peu d'effets en cycle trois.

5 Ainsi, l'étude spécifique réalisée à la demande de l'observatoire National de la Lecture, suite aux résultats des évaluations nationales annuelles en français, montre que parmi les élèves en grande difficulté de lecture, trois groupes peuvent être distingués : 4,3\% des élèves ont des grosses lacunes dans tous les domaines de la lecture; 7,8\% ont des difficultés principalement liées à leur lenteur dans l'exécution des tâches ; 2,8 \% ont des difficultés moindres (BAKTAVATSALOU, Pons, 1999). Notons que le premier groupe commet de nombreuses erreurs en phonologie mais ces performances augmentent au fur et à mesure lorsque l'on observe le dernier groupe constitué par les élèves en moins grande difficulté. Les performances en lecture (notamment) des élèves de CM2 ont été comparées entre 1987 et 2007 et font apparaître que si les résultats sont stables durant les dix premières années, une baisse significative des scores s'observe entre 1997 et 2007, chez les élèves les plus fragiles (ROCHER, 2008).

Les travaux sur la conscience phonologique concernent majoritairement le cycle deux et plus particulièrement la grande section. En revanche, les travaux sur les difficultés en lecture des élèves de cycle trois sont peu nombreux et portent majoritairement sur les difficultés de compréhension (NONNON, 2007).

6 En quoi l'entraînement de cette dernière peut-elle permettre à certains de ces élèves en grande difficulté de progresser et, par là même, de construire ou de reconstruire des éléments d'identité positive? En effet, au cycle 3, la maîtrise de la conscience phonologique est peu évaluée et, quant elle l'est, elle semble être largement influencée par le fait que les enseignants de ce cycle ne se sentent pas investis par cette tâche qu'ils estiment relever du cycle inférieur. De plus, lorsque la conscience phonologique est 
pratiquée au cycle 3 , son irrégularité liée à sa pratique nécessairement orale (l'écrit prédomine largement dans tous les domaines disciplinaires dès le CE2) ainsi que l'étude des éléments linguistiques tels que la syllabe et les phonèmes à l'antipode d'une culture littéraire...font qu'il est impossible d'en mesurer les effets sur les progrès des élèves.

\section{Cadre théorique}

7 Il se veut double en s'inscrivant, d'une part, dans la définition de la construction identitaire et, d'autre part, au cœur des recherches sur la conscience phonologique depuis les années soixante-dix.

L'articulation entre les deux se justifie par le fait que, les difficultés des élèves de cycle trois ou du collèges ont été considérées « soit dans une approche pédagogique englobante de l'échec scolaire en contextes scolaires difficiles, mettant l'accent sur la restauration de l'image de soi et du lien social, du rapport au travail et à l'avenir, du sens des apprentissages, soit dans une approche médicalisante, dans le contexte de l'enseignement spécialisé ou de la rééducation extrascolaire» (NONNON, 2007 : 7). D'autre part, poursuit l'auteur « il est vrai que des outils techniques inspirés de la psychologie cognitive ont été expérimentés et sont disponibles pour l'évaluation des difficultés de lecture comme pour des entrainements visant à y remédier, dans le domaine de la compréhension notamment. Cependant, sans discuter ici la question de leurs effets, ces entrainement reposent sur la perspective d'un travail intensif, spécialisé, décontextualisé par rapport au milieu et aux supports scolaires ordinaires. " (NONNON, $2007: 8$ ).

Ainsi, nous tentons ici cette articulation entre conscience phonologique et estime de soi en déplaçant à la fois le curseur temporel des difficultés en lecture (cycle 3 et non cycle 2) et le curseur spatial de l'estime de soi (dans la classe et non hors la classe).

\section{1. La construction identitaire et l'estime de soi}

8 L'ACELF$^{2}$ (Association Canadienne d'Education de Langue Française) définit la construction identitaire comme étant « un processus hautement dynamique au cours duquel la personne se définit et se reconnaît par sa façon de réfléchir, d'agir et de vouloir dans les contextes sociaux et l'environnement naturel où elle évolue ». Et d'ajouter que la famille, l'école... partagent leur responsabilité dans la construction identitaire des jeunes apprenants en les accompagnant. Nous voyons, concernant la lecture et la réussite ou l'échec de son apprentissage, combien chaque mot de la définition pèse de tout son poids sur la construction d'une identité positive ou négative.

La socialisation inscrit chaque être humain dans une société possédant ses codes, ses règles. La famille contribue à façonner cette identité au regard du milieu social d'appartenance, du lieu de vie, du cercle relationnel... La première identité se construit le plus couramment grâce à la fréquentation régulière de l'école. Cette dernière n'est pas choisie mais est très dépendante de facteurs eux-mêmes élus par les parents ou qui s'imposent à eux (culture, lieu(x) d'habitation, profession, religion...). L'élève va ainsi se construire à travers toutes les relations aux autres, l'identification à autrui. Comme le précise Gohier (2006, p. 153), le rapport à l'autre est constitué de retours sur soi favorisant la distanciation par rapport à l'autre mais aussi la reconnaissance de soi, de ses compétences et de ses manques. "Rapport à l'autre et rapport à soi sont deux mouvements incontournables d'une construction identitaire qui n'est pas que le reflet de l'autre ou enfermement sur soi ». 
9 L'identité renvoie aux divers sentiments d'appartenance sociale, en analysant les trajectoires à la fois collectives et individuelles, dans une double dimension, les transformations successives et leurs multiples formes. SAINSAULIEU (1977 : 319) définit le concept comme recouvrant le champ des rapports humains où «le sujet s'efforce d'opérer une synthèse entre les forces internes de son action, entre ce qu'il est pour lui et ce qu'il est pour les autres ». Il a ainsi défini des types d'identité au travail, parmi les conduites ouvrières tout particulièrement : l'identité fusionnelle, de retrait, de négociation et affinitaire. A la suite, DUBAR (1994) propose quatre autres types d'identité : l'identité d'exclusion, bloquée, de négociation individualisée et affinitaire. Son modèle des identités au travail s'appuie sur les processus biographiques (l'identité pour soi) et les mécanismes de reconnaissance (l'identité pour autrui). L'axe temporel renvoie aux discours de trajectoires continues et de ruptures. L'axe spatial renvoie à la reconnaissance ou non par les pairs, la hiérarchie... Le concept d'identité au travail nous semble intéressant pour l'école dans le sens où il est aussi au coeur des représentations dans le système de valeurs des élèves.

Récemment, KAISER et JENDOUBI (2009), analysent le concept de soi comme étant composé de l'estime de soi qui en serait le côté évaluatif et l'image de soi qui en serait la description. Ainsi, de par sa dimension affective, l'estime de soi ne renseigne pas sur les qualités et compétences réelles d'une personne mais plutôt sur la manière dont elle les ressent émotionnellement. L'estime de soi peut donc être évaluée individuellement ou collectivement au regard des caractéristiques des groupes sociaux auxquels la personne s'identifie (LUHTANEN, CROCKER, 1991 cité par BOUHRIS et LEYENS, 1998). Le développement d'un enfant se faisant dans les milieux familiaux, sociaux et scolaires, Cooley (1998) considère que les interactions sociales et linguistiques modèlent l'estime de soi dès la naissance. Les adultes proches (parents, enseignants) jouent alors un rôle déterminant dans la construction de l'intériorisation des jugements de valeurs que l'enfant construit, donnant ainsi naissance à l'estime de soi. Comme JENDOUBI (2002), nous retenons l'expression "estime de soi » plutôt qu' » image de soi »; sa valeur évaluative adhérant bien au monde scolaire qui nous préoccupe ici.

L'estime de soi se façonnerait donc au contact de différents environnements que l'enfant fréquente : familial, scolaire et social. L'image que renvoient les autres (qui appartiennent à ces trois sphères principales) à propos de ses compétences, son apparence, sa façon d'être...donne à l'enfant des informations sur l'opinion qu'ils ont de lui. Selon Cooley, c'est l'intériorisation de ces opinions qui favoriserait l'émergence de l'estime de soi. Cependant, elle serait fortement corrélée aux diverses interprétations que l'enfant perçoit en retour (JENDOUBI, 2002).

HARTER (1998) considère que c'est vers l'âge de 7-8 ans que l'enfant a une représentation globale de lui-même, mesurable. Avant cet âge, il a une idée de ses compétences scolaires notamment mais pas exclusivement, cependant les frontières entre ces divers domaines de compétences font qu'il ne s'est pas encore construit une valeur en tant que personne. De cette valeur globale dépendrait pourtant un facteur influençant son propre jugement : la variabilité de l'estime de soi selon les évènements traversés.

L'école représente un lieu d'interactions et va influer sur le développement de l'estime de soi. Les résultats scolaires jouent à cet égard un rôle important tant la réussite scolaire est liée, pour bon nombre de personnes, à la réussite sociale future.

Enfin, les difficultés en lecture au cycle 3 sont abordées de différentes façons. NAVARRO et LE DEUN (2004) faisant le lien entre théorie et pratique, préconisent la lecture d'une œuvre littéraire dans son entier afin de motiver l'élève, de développer les opérations mentales favorisant l'acte de lire, de changer sa représentation de la lecture mais aussi 
d'impulser la construction identitaire du sujet. Ces travaux constituent une référence importance car peu développée en ce sens. Celle-ci nous permet de faire le lien avec la compétence également mobilisable qui résulte des travaux scientifiques présentés ciaprès.

\section{2. La conscience phonologique}

Elle permet d'identifier les différentes composantes phonologiques des unités linguistiques (syllabes, rimes, phonèmes) et de les manipuler de manière tout à fait intentionnelle (LIBERMAN, 1993). Il y a consensus dans le monde scientifique pour affirmer que le niveau de conscience phonologique est un prédicteur d'un apprentissage de la lecture réussi ; que ces travaux soient réalisés auprès d'élèves maîtrisant la langue anglaise (STANOVICH et al., 1984; TUMNER, 1989) ou la langue française (MORAIS, ALEGRIA, CONTENT, 1987; ECALLE, 2000). Enfin, d'autres langues ont également été convoquées (Italien, Allemand, portugais...) apportant les mêmes résultats ${ }^{3}$.

A contrario, les élèves en difficulté (ainsi que les dyslexiques) ont, en règle générale, un déficit phonologique important (BRADLEY, BRYANT, 1978; SHANKWEILER, 1999). Ces recherches ont également révélé que l'entraînement de la conscience phonologique était possible et augmentait les capacités de lecture, notamment celles relevant du décodage, de l'orthographe et de l'écriture. Ces travaux sont peu nombreux en français (GOMBERT, 1990 ; LECOCQ, 1991), plus largement réalisés dans les milieux anglo-saxons (EHRI et al, 2001).

Cependant, un désaccord demeure quant aux unités linguistiques les plus importantes à travailler afin d'optimiser la réussite en lecture. Pour certains chercheurs, la conscience rimique serait fondamentale (GOSWAMI et BRYANT, 1990; GOSWAMI, 1993). La conscience syllabique se développe plus tôt que la conscience phonémique, plus difficile à acquérir (LIBERMAN, SHANKWEILER et al, 1974). Mais la conscience des phonèmes serait la plus importante pour la compréhension du principe alphabétique (SPRENGERCHAROLLES, COLE, 2003).

Bien que l'ensemble de ces recherches soit conséquent, certains auteurs émettent encore des doutes quant au lien entre conscience phonémique et capacités en lecture, argumentant en faveur de critères multiples impliqués dans son apprentissage. Ceci étant, il semble qu'un entraînement de la conscience phonémique, adjoint à l'apprentissage du nom et du son des lettres, soit la meilleure association afin de rendre l'apprentissage de la lecture plus performant, notamment le décodage (GENTAZ, COLE, BARA, 2003).

Enfin, notons que la très grande majorité des travaux scientifiques sur ce sujet concernent les élèves d'âge préscolaire et en apprentissage initial de la lecture.

\section{Méthodologie}

Si les données sont nombreuses concernant la période initiale de l'apprentissage de la lecture, peu sont disponibles concernant les difficultés des mauvais lecteurs du cycle trois ${ }^{4}$.

Notre recherche a consisté, dans un premier temps, à évaluer individuellement le niveau de conscience phonologique de 446 élèves de cycle 3 (CE2, CM1, CM2) répartis au sein de 21 classes appartenant à 5 écoles dont 4 en ZEP. Plusieurs de ces écoles étaient auparavant 
inscrites dans le Plan de Lutte contre l'Illettrisme.

Les notions de rimes, syllabes, phonèmes et opérations plus complexes de suppression, fusion ... de syllabes et de phonèmes ont été évaluées au début de l'année scolaire 2007-08; ainsi que la notion de mot qui, bien que ne faisant pas partie de la conscience phonologique, est un bon indicateur du niveau de représentation mentale du langage (annexe 1).

La passation, dévolue aux enseignants, a été réalisée à partir d'un enregistrement; la voix, les consignes et leur rappel, les exemples donnés, la vitesse ... étaient ainsi identiques afin de gommer autant que possible l'effet maître. Cependant, lorsque cela était nécessaire pour certains enfants, les professeurs des écoles avaient la possibilité de suspendre le déroulement de cet enregistrement, afin d'octroyer un temps de réflexion supplémentaire ; la vérification de la maitrise réelle de la compétence à ce niveau étant plus importante que le temps mis pour répondre.

Pour chaque élève, le taux de réussite aux huit items a été relevé. Dans un second temps, les cases ainsi renseignées ont été grisées lorsque l'enfant avait échoué totalement ou partiellement. En effet, l'évaluation demandée correspondant à un niveau de fin de CE1, l'exigence a reposé sur une maîtrise totale des items vérifiés; exceptions faites de la scansion ou non de la syllabe terminale et de la fusion de 3 syllabes dans le désordre, items pour lesquels un seuil d'erreurs était toléré.

Ensuite, chaque enseignant a effectué un entraînement en conscience phonologique régulier et identique pour l'ensemble des maîtres. Cet entrâ̂nement avait lieu deux fois par semaine, chaque séance durant vingt minutes. Il a été réalisé en une année pour trois écoles et en deux ans pour les autres. Nous avons élaboré le contenu des séquences et la progression à partir des résultats globaux de cette première évaluation. Ainsi, à partir d'un travail sur ce que les élèves connaissaient bien (les syllabes), les rimes ont été abordées puis plus largement les phonèmes dans l'ordre de fréquence d'apparition dans la langue française. Ce travail s'est inscrit dans un cadre ludique mais surtout collectif c'est-à-dire sans distinction entre élèves, tous adhérant à l'entraînement.

Enfin, les 267 élèves toujours inscrits dans ces écoles (c'est-à-dire soustraction faite de ceux ayant déménagé et/ou inscrits au collège) ont passé une seconde évaluation fin 2008-2009. La nature des épreuves était identique mais, bien entendu, les contenus différents. Lorsque les résultats ont été établis pour chaque élève à propos des deux évaluations, il a été demandé aux enseignants de les examiner afin de préciser si, en cas de progrès, ils avaient eux-mêmes constaté un niveau de lecture globalement plus performant, et inversement.

\section{Résultats}

Ils ont fait l'objet d'une correction individuelle. Pour chacun des huit items, un élève s'est vu attribuer un résultat. Puis, un codage a permis de faire ressortir trois catégories d'élèves :

- gris foncé : élève ayant échoué à 5, 6, 7 items ou à la totalité. Ils constituent le groupe des élèves en "grande difficulté ".

- blanc : élève ayant échoué à 3 ou 4 items. Cette catégorie constitue celle des élèves "moyens"

- gris clair : élève ayant échoué à un seul item ou ayant réussi l'ensemble. Cette dernière constitue les élèves d'un « bon niveau ». 


\section{V. Évaluation initiale} enfants qui n'ont pas compris la notion de mot et surtout la notion de rime à l'oral. Les opérations sur les syllabes sont presque totalement réussies et maîtrisées. La scansion ne pose pas de difficultés ; mais la fusion engendre des non-réponses, y compris lorsque les syllabes sont présentées dans l'ordre et entrecoupées par un bref silence. Les profils de classes révèlent un échec massif lorsqu'il s'agit de trouver le nombre de mots composant une phrase et de proposer des mots rimant avec d'autres (annexe 2). L'échec est moindre concernant les manipulations liées aux phonèmes mais les résultats en cas d'échec sont fortement associés au manque de construction des concepts de mot et de rime à l'oral. Enfin, c'est l'opération consistant à supprimer le phonème terminal qui révèle un grand nombre de confusions entre phonème et syllabe.

D'autres profils de classes et d'élèves se traduisent par une meilleure maîtrise de l'ensemble et montrent que lorsque l'entraînement a perduré au-delà du cycle 2, les élèves ne sont pas en échec scolaire, y compris lorsque l'on compare les résultats de différentes classes appartenant à une même école. Il semble donc que ce premier prédicteur non acquis en fin de maternelle ne le soit pas plus en cycle trois et que la scolarisation n'ait pas permis à elle seule de construire cette compétence indispensable si aucun entraînement régulier n'est poursuivi pour ces élèves.

\section{VI. Évaluation terminale}

Pour chaque classe restante, les profils ont été établis pour permettre de visualiser les résultats des élèves aux deux évaluations (annexe 3).

Nous nous sommes intéressée aux résultats des élèves qui étaient en grande difficulté lors de la première évaluation, c'est-à-dire ceux qui avaient échoué à plus de quatre items sur les huit proposés. Ils étaient 36 lors de l'évaluation initiale et 25 après l'entraînement. Les progrès sont visibles soit dans les opérations concernant les syllabes, soit dans les opérations concernant les rimes ; l'échec aux exercices mobilisant les phonèmes demeure presque toujours. Pour ceux qui étaient en difficulté mais qui avaient validé deux ou trois items sur les huit, les progrès sont également visibles. Cependant, plusieurs cas peuvent être répertoriés :

- une meilleure réussite (moins d'items échoués)

- un nombre d'items réussis constants mais avec déplacement: des élèves ayant réussi aux exercices concernant les syllabes et échoué à ceux impliquant les rimes et les phonèmes, voient leurs résultats progresser en rimes mais des erreurs se révèlent quant aux syllabes.

- des résultats moins bons lors de la seconde évaluation.

D'une manière générale, $4,12 \%$ des élèves en grande difficulté (gris foncé) sont sortis de cette catégorie. 
Tableau 1 Capital émotionnel : Différences Filles-Garçons

\begin{tabular}{|c|c|c|c|c|}
\hline $\begin{array}{c}\text { TOTAL } \\
\text { Circonscription }\end{array}$ & & & & Total \\
\hline $2007-08$ & $\begin{array}{r}36 \\
13,48 \%\end{array}$ & $\begin{array}{c}145 \\
54,31 \%\end{array}$ & $\begin{array}{r}86 \\
32,21 \%\end{array}$ & $\begin{array}{r}267 \\
100 \%\end{array}$ \\
\hline $2008-09$ & 25 & $\begin{array}{r}141 \\
52,81 \%\end{array}$ & $\begin{array}{r}101 \\
37,83 \%\end{array}$ & $\begin{array}{r}267 \\
100 \%\end{array}$ \\
\hline & $9,36 \%$ & -4 & +15 & \\
& -11 & $1,5 \%$ & $+5,62 \%$ & \\
\hline
\end{tabular}

\section{Discussion}

17 Il nous importe, dans le cadre de cet article, de discuter les résultats au regard de la construction identitaire des élèves. Pour ce faire, nous convoquons les propos des maîtres recueillis après présentation à chacun des résultats de la première évaluation, mais surtout, ceux recueillis lors de la présentation des résultats comparatifs, après l'entraînement.

Lorsque nous avons fait état des résultats de chaque élève après la première évaluation, il a été demandé à chaque enseignant de comparer le niveau ainsi obtenu (élève en difficulté ou élève moyen ou bon élève) avec le niveau global en lecture de ce même élève, c'est-à-dire sans distinction entre le décodage, la compréhension, le lexique, l'orthographe, l'écriture, la maîtrise de la langue orale... Sur les 18 classes représentées au début de l'année scolaire 2007-2008, 17 professeurs des écoles ont déclaré que les profils des élèves (de la classe plus largement) étaient conformes au niveau qu'eux-mêmes avaient relevé grâce aux diverses évaluations disponibles et cela quel que soit le niveau considéré (CE2-CM1-CM2), quelle que soit l'école et quel que soit l'enseignant. Seul un professeur des écoles de CM2 (ne pouvant donc pas réaliser l'entraînement futur) a émis des réserves quant à des résultats différents chez certains élèves.

Il semble donc que dans le cadre de cette recherche, l'évaluation en conscience phonologique soit un bon indicateur du niveau en lecture d'un élève. En effet nous pouvions, en amont de ces travaux, émettre l'hypothèse qu'un élève de cycle 3 ayant uniquement des difficultés de compréhension puisse révéler un bon niveau en conscience phonologique. Il n'en est rien. Les difficultés en lecture, quelle que soit leur nature, semblent avoir un impact sur la réussite ou non à une évaluation des compétences métaphonologiques.

En nous appuyant sur la définition de l'estime de soi de KAISER et JENDOUBI (2009), sur la notion de mesure de cette dernière (LUHTANEN, CROCKER, 1991 ; HARTER, 1998) et sur le rôle des interactions sociales et linguistiques dont les enseignants sont les principaux maîtres d'œuvre (COOLEY, 1998), nous pouvons dégager de cette recherche-action des hypothèses et idées-forces quant à la construction identitaire d'élèves de cycle 3 en grande difficulté de lecture.

- Si les élèves se construisent une valeur en tant que personnes vers 7-8 ans, nous pouvons supposer que ceux formant notre échantillon de départ, dont les plus jeunes étaient au CE2 lors de la première évaluation, se sont forgés une estime d'eux-mêmes dont ils sont conscients. S'il est possible d'évaluer l'estime de soi individuellement ou collectivement 
(nous nous appuyons pour cela sur la définition de JENDOUBI, 2002:9: «Quant à l'estime de soi, elle est généralement définie comme l'évaluation globale de la valeur de soi en tant que personne, c'est-à-dire le degré de satisfaction de soi-même »), nous pouvons supposer que pour ce dernier cas, le recueil des propos des enseignants lorsqu'ils commentent l'évolution des résultats des élèves en conscience phonologique, peuvent constituer un matériau exploitable quant à cette dimension collective, d'autant que deux facteurs dédouanent les professeurs des écoles d'un exposé subjectif. Le premier est qu'ils ignorent que la dimension identitaire des élèves sera analysée à travers leurs propos ; ils ont comme unique consigne lors de l'entretien de commenter les progrès ou non en lecture des élèves. Le second est que les élèves étant suivis pendant deux ans, ceux-ci ont donc changé de classe et par voie de conséquence, de maîtres. Ces derniers ne donnent donc pas uniquement leur avis sur leurs élèves mais surtout et principalement sur les élèves de leurs collègues l'année précédente. Ce n'en est pas moins subjectif (ce qui n'est pas négatif de mon point de vue !) pour autant ...

- Si nous nous référons à la recherche de Perron (1991), l'assouplissement des exigences des enseignants quant aux difficultés des élèves, favorise chez ces derniers une amélioration de leur estime d'eux-mêmes. L'enfant s'adapte plus facilement à un apprentissage nouveau si tant est qu'il se sent capable au début de cet apprentissage de le réussir. L'entrainement en conscience phonologique dispensé à toute la classe a permis de ne pas focaliser un apprentissage exclusivement destiné à un groupe en difficultés. Cet entraînement, dont le point de départ (les syllabes) constitue une compétence déjà maîtrisée par la très grande majorité, permet d'installer la confiance entre l'enseignant et ses élèves. L'apprentissage progressif en boucle, c'est-à-dire reprenant les exercices déjà réalisés les séances antérieures, avec intégration a minima d'une notion nouvelle, favorise la pérennité de cette confiance et la valorisation des réussites obligatoirement plus nombreuses que les échecs.

- Les professeurs des écoles ont transcrit eux-mêmes, pour chaque élève, leurs réactions, observations quant à la comparaison des résultats des élèves aux deux évaluations en conscience phonologique et leur propre connaissance des progrès des élèves depuis une année voire deux. Il ressort de ces transcriptions que les maîtres qui ont mentionné des observations en termes de confiance en soi, persévérance, concentration...et progrès en lecture (versus manque de confiance en soi, problèmes familiaux...difficultés persistantes en lecture...), sont les maîtres qui ont poursuivi spontanément l'entraînement deux années. A contrario, les maîtres ayant mis en place cet entraînement une seule année, ne renseignent la grille qu'en termes de progrès ou difficultés en lecture à voix haute, compréhension, orthographe. Ainsi, dans le premier cas, nous pouvons citer des annotations comme : «Bonne élève, trop confiante en elle "; "Gros progrès généraux. Lecture et compréhension correctes. Plus à l'aise et meilleure confiance en elle »; "Manque de confiance, stressée par l'épreuve. Lecture et compréhension correctes à l'écrit, quelques confusions de sons persistent. »; Dans le second cas, les annotations sont plus brèves, et exclusivement exprimées en termes de réussite ou d'échec: "Très en difficulté"; " Très bonne élève »; "Grosses difficultés en orthographe, très bon en vocabulaire et à l'oral »; « Elève qui a encore des difficultés à voix haute mais qui progresse »; « Elève en grande difficulté mais en phase de progrès ». Nous pouvons donc supposer, en nous appuyant sur la nature de leurs propos, que les premiers sont autant en attente, chez les élèves, de progrès en lecture que d'épanouissement personnel. A contrario, nous pouvons supposer que les seconds sont des enseignants qui sont davantage en attente de progrès en lecture chez les apprenants. Nous nous référons également à la nature de leurs propos, plus concis et exclusivement axés sur la maîtrise disciplinaire ; ce qui peut, par voie de conséquence, expliquer leur plus court investissement dans cet entraînement. 


\begin{abstract}
- Nous l'avons précisé en introduction, l'oral est beaucoup moins pratiqué au cycle 3 ; l'écrit prédominant bon nombre d'enseignements/apprentissages disciplinaires à ce niveau. L'entraînement en conscience phonologique étant exclusivement à l'oral, certains maîtres ont eu l'impression de « perdre du temps » voire le sentiment de «ne pas avoir réellement travaillé » avec leurs élèves ; la preuve par la trace écrite étant complètement absente. Faire le deuil de la trace écrite représente pour certains professeurs des écoles un réel effort vis-àvis de la hiérarchie, des parents...Nous touchons là, la propre estime des enseignants et l'impossible reconnaissance de la valeur de leur travail sans cette trace écrite. Cependant, côté élèves, les interactions orales font que la réflexion de chacun, sa pensée, ses choix, ses solutions et leurs démonstrations...sont transmises et donc partagées entre tous les élèves et cela de façon récurrente, régulière. Cela n'est pas le cas, en production écrite par exemple lorsque chacun est face à sa feuille et transmet au bout d'un certain temps ce qui ne demeure qu'un produit fini. Aucune interaction orale ne permet à chaque élève de savoir comment ses camarades en sont arrivés à produire leur texte. En cela, un enseignement réalisé exclusivement à l'oral repose sur des apprentissages disciplinaires précis mais aussi sur l'apprentissage et le partage entre élèves de démarches, de modes de réflexion...que l'écrit ne laisse jamais entrevoir. Cet entraînement en conscience phonologique a certainement permis à des élèves de constater que d'autres avaient les mêmes difficultés ; que certains avaient des démarches de résolutions dont eux-mêmes ignoraient l'existence ... et surtout que progresser était possible.
\end{abstract}

\title{
VIII. Conclusion
}

19 Cette recherche, ayant un objectif précis quant à la mesure des progrès en lecture des élèves, n'avait pas comme objectif second l'évaluation par les élèves eux-mêmes de leur épanouissement personnel. Néanmoins, à travers les écrits de leurs enseignants, nous pouvons faire état ou non de celui-ci. Ainsi, même si comme le rappelle KAISER et JENDOUBI $(2009$, p. 8) «Si l'école, et plus particulièrement le jugement des enseignants, influence l'estime de soi, la manière dont les élèves se perçoivent agit également sur leur attitude face aux apprentissages", nous pouvons ajouter que tous les enseignants, inconsciemment, ne positionnent pas forcément sur le même plan la réussite aux apprentissages et l'épanouissement de l'élève.

La recherche de SCHUNK (2003) sur la compréhension en lecture illustre ces propos. L'auteur montre combien la réussite des élèves est meilleure lorsque les enseignants enseignent des stratégies consistant à l'identifier les principales idées d'un texte; cette réussite s'accompagnant d'une meilleure confiance en soi des apprenants. Les résultats sont moins bons lorsqu'il s'agit pour eux de répondre uniquement à des questions de compréhension.

S'agissant d'un apprentissage aussi fondamental que la lecture, il semble bien que l'enseignement de stratégies allié à l'enseignement d'un contenu disciplinaire soit la meilleure association pour que les élèves progressent tout en s'épanouissement dans cette microsociété qu'est l'école. 
Anne xe 1

Item 1: notion de mot : dénombrement (3 phrases de moins de six mots).

Items 2 et 3: notion de fime : association mot rime et association mot rime plus complex

Ane X8 2: Ecole 5 classe 4 (CES2)

\begin{tabular}{|c|c|c|c|c|c|c|c|c|c|}
\hline $\begin{array}{l}\text { liems } \\
\text { Eleve es }\end{array}$ & Mots & rimes & $\begin{array}{c}3 \\
\text { rimes } 5\end{array}$ & ${ }^{4} 4$ & $\begin{array}{l}5 \\
\text { syllabes }\end{array}$ & $\begin{array}{c}5 \\
\text { syllabes }\end{array}$ & $\begin{array}{c}7 \\
\text { phonemes }\end{array}$ & $\begin{array}{c}\text { phè } \\
\text { phonèmes }\end{array}$ & Bilan \\
\hline AMZO & $3 / 3$ & 212 & 3/3 & $5 / 6$ & $5 / 5$ & $4 / 5$ & $3 / 3$ & $4 / 4$ & \\
\hline BASH & $0 \nmid 3$ & $2 / 2$ & $3 / 3$ & $4 / 6$ & $4 / 5$ & $4 / 5$ & $2 / 3$ & $2 / 4$ & \\
\hline BAM E & $2 / 3$ & $2 / 2$ & $3 / 3$ & $6 / 6$ & $5 / 5$ & $a / 5$ & $3 / 3$ & 0,14 & \\
\hline BEZE & $2 / 3$ & $0 / 2$ & $1 / 3$ & $5 / 6$ & $4 / 5$ & $4 / 5$ & $3 / 3$ & $4 / 4$ & \\
\hline CHS A & 3/3 & 012 & $1 / 3$ & $4 / 6$ & $2 / 5$ & 315 & $3 / 3$ & $4 / 4$ & \\
\hline ELSA & $2 / 3$ & 212 & 0,3 & $5 / 6$ & $4 / 5$ & $5 / 5$ & $2 / 3$ & $3 / 4$ & \\
\hline FECA & $1 / 3$ & $0 / 2$ & $0 / 3$ & $1 / 6$ & $2 / 5$ & 0,5 & $3 / 3$ & $0 / 4$ & \\
\hline GHSA & $2 / 3$ & $2 / 3$ & 3/3 & $5 / 6$ & $4 / 5$ & $5 / 5$ & $3 / 3$ & $2 / 4$ & \\
\hline HAAN & $0 / 3$ & $0 / 2$ & 113 & $5 / 5$ & $5 / 5$ & $2 / 5$ & $3 / 3$ & $3 / 4$ & \\
\hline HOJU & 3/3 & $0 / 2$ & 0,3 & $6 / 6$ & $5 / 5$ & $5 / 5$ & $2 / 3$ & $4 / 4$ & \\
\hline KAN U & 3/3 & $2 / 2$ & $1 / 3$ & $6 / 6$ & $4 / 5$ & $4 / 5$ & $3 / 3$ & $2 / 4$ & \\
\hline KHCE & $3 / 3$ & $1 / 2$ & $3 / 3$ & $5 / 6$ & $5 / 5$ & $2 / 5$ & $3 / 3$ & 0,4 & \\
\hline LAHU & $0 / 3$ & $1 / 2$ & $1 / 3$ & $5 / 6$ & $4 / 5$ & $3 / 5$ & $3 / 3$ & $2 / 4$ & \\
\hline LESO & $3 / 3$ & $2 / 2$ & $1 / 3$ & $5 / 6$ & $4 / 5$ & $\alpha, 5$ & $3 / 3$ & $2 / 4$ & \\
\hline LECO & $2 / 3$ & 112 & $1 / 3$ & $5 / 6$ & $4 / 5$ & $Q 5$ & $3 / 3$ & 214 & \\
\hline MAEN & $0 / 3$ & 212 & $2 / 3$ & $6 / 6$ & $4 / 5$ & $4 / 5$ & $3 / 3$ & $2 / 4$ & \\
\hline MAJu & $3 / 3$ & 212 & 3/3 & $6 / 6$ & $4 / 5$ & $5 / 5$ & $3 / 3$ & $4 / 4$ & \\
\hline MOFL. & $2 / 3$ & 222 & $0 / 3$ & $5 / 6$ & $4 / 5$ & 25 & $3 / 3$ & $4 / 4$ & \\
\hline ONES & $3 / 3$ & 012 & 013 & $5 / 6$ & $4 / 5$ & 315 & $3 / 3$ & $2 / 4$ & \\
\hline SAVA & 0,3 & $2 / 2$ & $2 / 3$ & $4 / 6$ & $3 / 5$ & 255 & $3 / 3$ & $4 / 4$ & \\
\hline UMOI & $3 / 3$ & $2 / 2$ & $3 / 3$ & $6 / 6$ & $4 / 5$ & 4,5 & $3 / 3$ & $3 / 4$ & \\
\hline VIAL & $\alpha / 3$ & $1 / 2$ & $2 / 3$ & $5 / 6$ & $3 / 5$ & $3 / 5$ & $3 / 3$ & $4 / 4$ & \\
\hline ZEEL & $2 / 3$ & $0 / 2$ & $0 / 3$ & $5 / 6$ & $3 / 5$ & $1 / 5$ & $1 / 3$ & $4 / 4$ & \\
\hline
\end{tabular}

Légende : les cases iterns gris foncé dans le tableau signifient que lélève a échoué

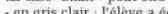

(n)

- en gris foncé: l'élève a échoué a $5,6.7$ ou

Anne Xe 3 : Ecole 4 classe CM1 (21 élèves)

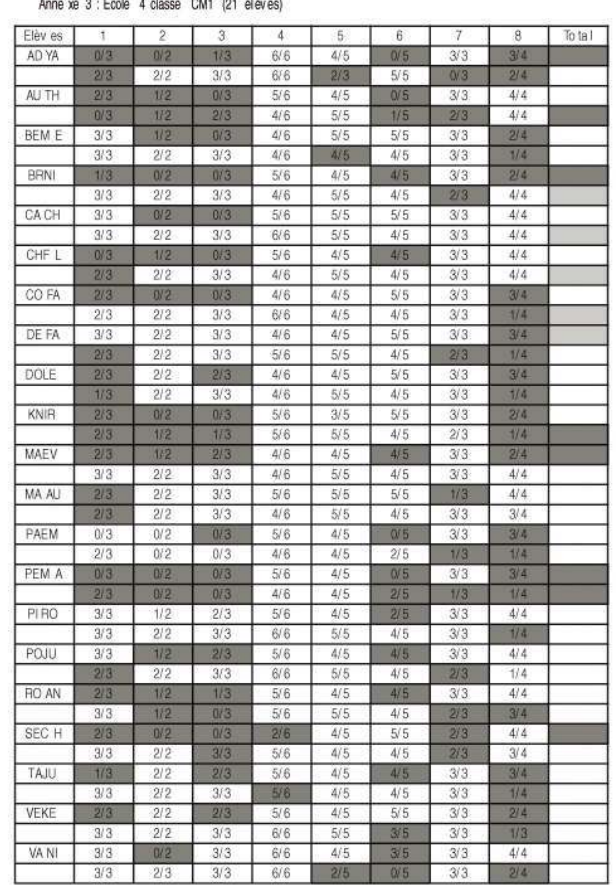

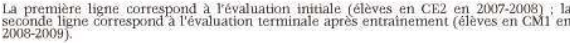




\section{BIBLIOGRAPHIE}

AYRAUd M., GUibert P. (2000). Constructions identitaires. In Blanchard-Laville C., Nadot S. (dir.).

Malaise dans la formation des enseignants. Paris : L'Harmattan, p. 233-265.

BAKTAVATSALOU R., PONS C. (1999). «Etude spécifique sur les élèves en difficulté en lecture à

l'entrée en sixième ", Note d'information, 48, Paris : MEN, DEPP.

BOURHIS R. Y., LEYENS J.P. (1998). Stéréotypes, discrimination et relations intergroupes. Wavre: Mardaga.

BRADLEY L., BRYANT P.E. (1978). Difficulties in auditory organization as a possible cause of reading

backwards. Nature, 271, p. 746-747.

BRADLEY L., BRYANT P.E. (1983). Categorising sounds and learning to read: A causal connection. Nature, 310 , p. 419-421.

COOLEY C.H., Nature humaine et ordre social, la signification du « Je », in Bolognini M., Preteur Y. (1998). Estime de soi, perspectives développementales. Lausanne : Delachaux et Niestlé.

DUBAR C. (1994). Identités collectives et individuelles dans le champ professionnel. Traité de sociologie du travail. Bruxelles : De Boeck.

ECALLE J., (2000). Prédiction de réussite scolaire en lecture-écriture au cycle II. Revue européenne de Psychologie appliquée, 50, p. 81-85.

EHRI L. C., NUNES S.R., WILLOWS D.M., et AL (2001). Phonemic awareness instruction helps children learn to read: evidence from the National reading Panel's meta-analysis. Reading Research Quaterly , 36, p. 250-287.

GENTAZ E., COLÉ P., BARA F. (2003). Evaluation d'entraînements multi-sensoriels de préparation à la lecture chez les jeunes enfants de grande section de maternelle : étude sur la contribution du système haptique manuel. L'année Psychologique, 104, p. 561-584.

GOHIER C. (2006). Education et fragmentation identitaire : à la recherche d'un centre de gravité. Education et Francophonie, Vol XXXIV : 1, p. 148-161.

GOMBERT J.E. (1990). Le développement métalinguistique. Paris: PUF.

GOSWAMI U., BRYANT P. (1990). Phonology skill and learning to read. Hillsdale N. J.: Erlbaum.

GOSWAMI U. (1993). Toward an interactive analogy model of reading development: decoding wowel graphemes in beginning reading. Journal of experimental Child Psychology, 56, p. 443-475.

HARTER S. (1998). Comprendre l'estime de soi de l'enfant et de l'adolescent : considérations historiques et méthodologiques. In M. Bolognini, Y. Prêteur (Dir.). Estime de soi, perspectives développementales. Lausanne : Delachaux et Niestlé, p. 57-81.

JENDOUBI V. (2002). Estime de soi et éducation scolaire. Genève : SRED.

KAISER A., JENDOUBI V. (2009). La perception de soi. Comment les élèves de 5e/6e primaire voient leurs compétences et ce qu'en pensent leurs enseignant-e-s. Genève : SRED.

LECOCQ P. (1991). Apprentissage de la lecture et dyslexie. Liège : Mardaga. 
LIBERMAN I. Y. (1993). Segmentation of the spoken word and reading acquisition. Bulletin of the Orton Society, 23, p. 65-77.

LIBERMAN I. Y., SHANKWEILER D. et al. (1974). Explicite syllable and phoneme segmentation in the young child. Journal of Experimental Child Psychology, 18 (2), p. 201-212.

LUHTANEN R., CROCKER J. (1991). Self-esteem and intergroup comparisons: Toward a theory of collective self-esteem. In J. Suls and T. A. Wills (Eds.), Social comparison: Contemporary theory and researchp. Hillsdale, NJ: Lawrence Erlbaum, p. 211-234.

MORAIS J., ALEGRIA J., CONTENT A., (1987). The relationships between segmental analysis and alphabetic literacy: an interactive view. Cahiers de Psychologie Cognitive, 7, p. 415-438.

NAVARRO H., LE DEUN E. (2004). Prévenir l'illettrisme une autre approche pour reconstruire la lecture au cycle 3. Paris : Magnard.

NONNON E., (2007). L'école et ses « mauvais lecteurs ». Repères, 37, p. 5-30.

PERRON R. (1991) (Dir.). Les représentations de soi. Développements, dynamiques, conflits. Toulouse : Privat.

ROCHER T. (2008). « Lire, écrire, compter : les performances des élèves à vingt ans d'intervalle 1987-2007 », Note d'information, 38, Paris : MEN, DEPP.

SAINSAULIEU R. (1977). L'identité au travail. Les effets culturels de l'organisation.

Paris : Presses de la FNSP.

SCHUNK D. H. (2003). Self-efficacy for reading and writing: influence for modeling, goal setting and self-evaluation. Reading and Writing Quaterly, vol 19, 2, p. 159-172.

SHANKWEILER D. (1999). Words to meanings. Scientific Studies of Reading, 3, 113-127.

SPRENGER-CHAROLLES L., COLÉ P. (2003). Lecture et dyslexie. Approche cognitive. Paris: Dunod.

STANOVICH K.E., CUNNINGHAM A.E., CRAMER B.B. (1984). Assessing phonological awareness in kindergarten children: issues of task comparability. Journal of Experimental Child Psychology, 38, p. 175-190.

TUMNER W. E. (1989). Conscience phonologique et acquisition de la langue écrite. In Rieben L., Perfetti C. L'apprenti-lecteur : recherches empiriques et implications pédagogiques. Paris : Delachaux et Niestlé, p. 197-220.

\section{NOTES}

1. Nonnon E., Goigoux R. (coord.), (2007). Les ratés de l'apprentissage de la lecture à l'école et au collège. Repères, 35.

2. 62ème Congrès annuel

3. Pour une revue de la question, se reporter à Bara F., Gentaz E., Colé P. (2004). Les effets des entraînements phonologiques et multisensoriels destinés à favoriser l'apprentissage de la lecture chez les jeunes enfants ». Enfance, 4, vol. 56, p.387-403.

4. Revue Repères $n^{\circ} 35$ 


\section{RÉSUMÉS}

La société participe à construire l'identité d'un enfant. La socialisation de ce dernier intègre les caractéristiques locales, professionnelles, culturelles... de sa famille. Puis, ses expériences sociales vont contribuer à enrichir et transformer son identité. A l'école, ses performances scolaires, notamment celles réalisées dans et par l'apprentissage de la lecture, concourent également à construire, développer et modifier son identité en lien avec celle dont il a hérité. Lorsque cet apprentissage précis n'est pas ou peu acquis, cela provoque une remise en cause de l'identité en construction. La recherche que nous proposons d'exposer vise à analyser si une catégorie précise et délimitée de difficultés peut être repérée et identifiée comme étant un marqueur individuel et collectif important de l'échec persistant en lecture au cycle trois. Côté élève, il s'agit précisément, au-delà de l'approche globale de ses difficultés, de lui permettre de travailler conjointement sur la restauration de son image et du lien social sans différenciation des remédiations apportées au sein de la classe entière grâce à un entraînement de la conscience phonologique sur le long terme.

The society participates to build the identity of a child. His socialization integrates the local, professional, cultural characteristics of his family. Then, his social experiences are going to contribute to enrich and transform his identity. His school performances, in particular those realized in learning of reading, also contribute to build, to develop and to modify identity in connection with the one from which he inherited. When this learning is not or little acquired, it provokes a questioning of the identity under construction. The research which we suggest analyzes if a category by reading difficulties can be identified as being an important individual and collective marker of the failure persisting in reading in the French cycle three. It is exactly a question, beyond the global approach of his difficulties, of allowing him to work collectively on the restoration of its image and the social link without differentiation but with training of phonological awareness for a long time.

\section{INDEX}

Mots-clés : conscience phonologique, construction identitaire, cycle 3, difficulté, lecture

\section{AUTEUR}

\section{SOPHIE BRIQUET-DUHAZÉ}

IUFM de Haute-Normandie - Université de Rouen 